\title{
Implicaciones psicológicas de la esclerosis múltiple Un estudio preliminar
}

\author{
Jose Aguilar-Falomir \\ al362191@uji.es \\ Iván Álvarez Bardón \\ al364930@uji.es \\ María Carla Balmaseda-Álvarez \\ al364852@uji.es \\ Víctor Blasco lbáñez \\ al362166@uji.es \\ Beatriz Gil-Juliá \\ bgil@uji.es
}

\section{Resumen}

La esclerosis múltiple compromete la capacidad motora y cognitiva de los afectados, implicando afectación psicológica en diversas áreas de su vida. En este trabajo pretendemos evaluar la depresión, la ansiedad y la inadaptación de pacientes con esclerosis múltiple, así como analizar las posibles diferencias en función de distintas variables sociodemográficas.

La muestra está formada por 60 enfermos $(38,33 \%$ hombres y $61,67 \%$ mujeres $)$ con una edad media de 44,38 años (DT $=9,5$ ). Todos ellos han cumplimentado la escala de inadaptación (Echeburúa, Corral y Fernández Montalvo 2000) y la escala de ansiedad y depresión hospitalaria (HADS; Zigmond y Snaith 1983).

Los resultados muestran una media en ansiedad de $7,48(D T=3,62)$ y en depresión de 6,98 (DT =4,34). En cuanto a la escala de inadaptación, los pacientes presentan una interferencia global de 2,87 (DT =1,33) en un rango de $0-5$, siendo el ámbito más afectado el de "Trabajo y/o estudios» (media $=3,08$; $\mathrm{DT}=1,61)$ y el menos afectado el de "Vida familiar» (media $=1,72 ; \mathrm{DT}=1,38$ ). Los análisis muestran diferencias significativas en función del género en ansiedad $(t=2,61 ; \quad p$-valor $=0,013)$, depresión $(t=2,75 ; \quad p$-valor $=0,008) \quad e$ interferencia global $(t=1,93 ; p$-valor $=0,059)$. Las diferencias en función del género también son significativas en «Relación de pareja» $(t=2,26$; $p$ valor $=0,029$ ). Respecto a la situación laboral, se han obtenido diferencias en todas las áreas de interferencia, así como en la inadaptación global ( $p$ valor $=0,002$ ).

Estos datos evidencian afectación emocional en los pacientes, en línea con estudios anteriores, observándose diferencias en función de distintas variables, 
información valiosa para iniciar programas de prevención/intervención que mejoren el afrontamiento de la enfermedad.

Palabras clave: esclerosis múltiple, depresión, ansiedad, inadaptación, implicaciones psicológicas.

\section{Abstract}

Multiple Sclerosis alters motor and cognitive capacity, implying psychological affectation in diverse areas of their lives. The aim of this paper is to evaluate depression, anxiety and adjustment in patients who suffer from Multiple Sclerosis, and to analyze differences depending on several socio-demographic variables.

The sample includes 60 patients $(38.33 \%$ men and $61.67 \%$ women), whose average age is $44,38(S D=9,5)$. They have all completed the Unadaptability Scale (Echeburúa, Corral \& Fernández Montalvo 2000) and Hospital Anxiety and Depression Scale (HADS; Zigmond and Snaith 1983).

The results show a mean score of $7.48(S D=3.62)$ in anxiety, and 6.98 $(S D=4.34)$ in depression. Regarding Unadaptability Scale, the global score is of $2.87(\mathrm{SD}=1.33)$ in a $0-5$ rank. The most affected area is "Work and/or studies" (Mean $=3.08 ; S D=1.61$ ) and the least affected "Family life" (Mean $=1.72$; $S D=1.38$ ). Analysis show significant differences according to the gender in anxiety $(\mathrm{t}=2.61 ; \mathrm{p}$-value $=0.013)$, depression $(\mathrm{t}=2.75 ; \mathrm{p}$-value $=0.008)$ and global unadaptability $(t=1.93 ; p$-value $=0.059)$. Differences according to the gender are also significant in "Couple's relationship" $(t=2.26$; $p$-value $=0.029)$. Regarding employment status, differences in all unadaptability areas and in global unadaptability have been found ( $p$-value $=0.02$ )

These data evidence emotional disturbances in patients, in line with prior research, showing differences according to several variables. This represents valuable information to initiate prevention/intervention programmes to improve disease's coping.

Keywords: multiple sclerosis, depression, anxiety, unadaptability, psychological implications.

\section{Introducción}

La esclerosis múltiple (EM) es una enfermedad crónica, degenerativa y autoinmune del sistema nervioso central, que afecta al cerebro y a la médula espinal. Lesiona la vaina de mielina, el material que rodea y protege las células nerviosas (MedlinePlus 2017). En la actualidad, la padecen aproximadamente 2 millones de personas en el mundo. Está caracterizada por un inicio temprano, oscilando entre los 20-40 años de edad, pudiendo establecerse la media de la edad de aparición de los síntomas en $30,29 \pm 7,4$ años. Por otro lado, la edad del diagnóstico se sitúa en torno a los $34,29 \pm 8,2$ años, lo que supone una diferencia de tiempo de $4 \pm 3,2$ años entre las primeras manifestaciones de la enfermedad y el diagnóstico (Kidd et al. 2017; Arbinaga lbarzábal 2003).

Los síntomas que aparecen como consecuencia de la lesión pueden incluir: alteraciones de la vista, debilidad muscular, pérdida sensorial y motora, problemas con la coordinación y el equilibrio, sensaciones como entumecimiento, picazón o pinchazos, fatiga, dolor y problemas de deterioro cognitivo. 
Como consecuencia de todos estos síntomas, las personas que padecen EM se enfrentan a problemas de índole psicológica en su día a día, presentando depresión y ansiedad de manera más común que la población general (Kidd et al. 2017; Muñoz San José et al. 2015).

Diversos estudios recientes relacionan la depresión o la ansiedad asociada al diagnóstico de la EM con una peor evolución de la enfermedad. Además, se establece que estas afectan a más del $20 \%$ de la población con EM, siendo las tasas de prevalencia de depresión y ansiedad del $31 \%$ y del $22 \%$, respectivamente (Muñoz San José et al. 2015; Boeschoten 2016). Estos datos varían ligeramente entre diferentes estudios, estableciéndose una prevalencia del $36,6 \%$ de depresión en un estudio del 2015 en el que participaron 60 pacientes con EM (Rodrigues de Cerqueira, Semionato de Andrade, Godoy Barreiros, Teixeira y Nardi 2015). Es importante mencionar que, para medir el nivel de ansiedad y depresión de los pacientes, diversos estudios emplearon el HADS (Hospital Anxiety and Depression Scale; Zigmond y Snaith 1983) (Kidd et al. 2017; Boeschoten 2016).

En una revisión sistemática reciente sobre intervenciones en pacientes con EM (Kidd et al. 2017) se concluye que la terapia cognitivo-conductual mejora la calidad de vida de los pacientes, si bien se requiere de más investigación para determinar su efecto sobre la mejora en sintomatología de ansiedad y depresión, puesto que los resultados parecen variar a lo largo del tiempo. Además, se plantea que en un futuro se debe tener en cuenta la figura del cuidador y las nuevas tecnologías que facilitan el acceso a la terapia cognitivo-conductual a las personas con una mayor incapacidad motora. Asimismo, otras líneas de investigación proponen el mindfulness o atención plena como una intervención psicoterapéutica que mejora el estado emocional de los pacientes con EM (Muñoz San José et al. 2015).

A pesar del impacto emocional que puede tener una enfermedad como la EM, existe un gran vacío en la literatura revisada sobre la afectación emocional que conlleva y en cómo dicha afectación puede influir en el curso y el desarrollo de la enfermedad. Además de la escasez de información sobre el tema, los resultados encontrados en la literatura no parecen ser concluyentes y enfatizan la necesidad de una investigación más profunda al respecto (Kidd et al. 2017; Arbinaga Ibarzábal 2003; Muñoz San José et al. 2015; Boeschoten 2016; Rodrigues de Cerqueira, Semionato de Andrade, Godoy Barreiros, Teixeira y Nardi 2015). Por ello, el objetivo de este trabajo ha sido intentar conocer mejor la relación entre la afectación emocional y el desarrollo de la EM, a partir de la evaluación de los síntomas de depresión, ansiedad e inadaptación en pacientes con EM, teniendo en cuenta diversas variables sociodemográficas.

\section{Métodos}

\section{Sujetos}

El trabajo se ha realizado con 60 sujetos voluntarios $(38,33 \%$ hombres y $61,67 \%$ mujeres) diagnosticados con esclerosis múltiple, miembros de asociaciones de dicha enfermedad de diferentes comunidades autónomas españolas (Murcia, Aragón, Castilla y León, Comunidad Valenciana, Galicia, País Vasco e Islas Baleares) (véase la tabla 4). La edad media de la muestra global es de 44,38 (DT =9,50), siendo la de los hombres de 45,39 (DT = 8,64) y la de las mujeres de 43,76 (DT =10,06). Las principales características de la muestra aparecen en las tablas 1-4. 
Tabla 1

Análisis de los porcentajes del estado civil

\begin{tabular}{|c|c|}
\hline Estado civil & Porcentajes \\
\hline Casado/a & $65 \%$ \\
\hline Soltero/a & $15 \%$ \\
\hline $\begin{array}{c}\text { Soltero/a pero con } \\
\text { pareja estable }\end{array}$ & $0 \%$ \\
\hline Viudo/a & $1,67 \%$ \\
\hline Otros & $3,33 \%$ \\
\hline
\end{tabular}

Tabla 2

Porcentajes de la edad de aparición de los primeros sintomas

\begin{tabular}{|c|c|}
\hline Edad síntomas & Porcentajes \\
\hline \hline Niñez (0-12 años) & $3,33 \%$ \\
\hline Adolescencia (13-17 años) & $6,67 \%$ \\
\hline Juventud (18-34 años) & $61,67 \%$ \\
\hline Adultez (35 años o más) & $28,33 \%$ \\
\hline
\end{tabular}

Tabla 3

Porcentajes de la situación laboral

\begin{tabular}{|c|c|}
\hline Situación laboral & Porcentajes \\
\hline Empleado & $45,76 \%$ \\
\hline Desempleado & $10,17 \%$ \\
\hline Jubilado & $15,25 \%$ \\
\hline Incapacidad laboral & $23,73 \%$ \\
\hline Otros & $5,08 \%$ \\
\hline
\end{tabular}


Tabla 4

Porcentajes de la comunidad autónoma de residencia

\begin{tabular}{|c|c|}
\hline $\begin{array}{c}\text { Comunidad } \\
\text { autónoma }\end{array}$ & Porcentajes \\
\hline Murcia & $13,33 \%$ \\
\hline Castilla y León & $31,67 \%$ \\
\hline País Vasco & $1,67 \%$ \\
\hline Islas Baleares & $1,67 \%$ \\
\hline Galicia & $13,33 \%$ \\
\hline Aragón & $25 \%$ \\
\hline Comunidad & $13,33 \%$ \\
\hline Valenciana & \\
\hline
\end{tabular}

\section{Procedimiento}

El estudio está basado en una encuesta que fue enviada por correo electrónico a las asociaciones de las comunidades autónomas antes nombradas y a la que respondieron 60 sujetos.

Todos los participantes fueron informados de las características del estudio, así como de la confidencialidad y el anonimato de los datos recogidos. Tras firmar el consentimiento informado, los participantes contestaron libre y voluntariamente a los cuestionarios.

\section{Análisis estadísticos}

Los análisis estadísticos se han realizado mediante la importación del archivo Excel al programa $\mathrm{R}$, con el cual se han podido obtener los datos deseados. Se llevaron a cabo t de Student, ANOVA y tablas de contingencia chi cuadrado con un $95 \%$ de confianza para obtener las comparaciones de las medias.

\section{Instrumentos}

La encuesta enviada por correo electrónico incluye un apartado en el que se recogen datos sociodemográficos (sexo, edad, estado civil, situación laboral, comunidad autónoma y edad de aparición de los primeros síntomas) y otro bloque en el que se presentan dos herramientas de evaluación psicológica: la escala hospitalaria de ansiedad y depresión (HADS, Zigmond y Sanith 1983) (Zigmond y Snaith 1983) y la escala de inadaptación de Echeburúa Odriozola, De Corral Gargallo y FernándezMontalvo (2000).

La escala hospitalaria de ansiedad y depresión (HADS) (Zigmond y Snaith 1983) es frecuentemente utilizada por profesionales de la salud para medir el nivel de ansiedad y depresión de los pacientes. Está constituida por 14 ítems que valoran ambos aspectos mediante un sistema de puntuación que va de 0 a 3 en cada ítem. Estos 14 ítems se dividen en dos subcategorías según el carácter que evalúen (los pares miden la depresión y los impares, la ansiedad). Se suman los 7 ítems de cada subcategoría por separado. Los puntos de corte son: normal/ausencia de ansiedad-depresión entre 0 y 7 , dudoso/posible problema clínico entre 8 y 10 y probable problema clínico más de 10. 
La escala de inadaptación (EI) (Echeburúa Odriozola, De Corral Gargallo y FernándezMontalvo 2000) tiene como objetivo determinar el grado de desajuste que produce un determinado trastorno en la vida cotidiana, tanto en general como en áreas más concretas (trabajo o estudios, vida social, tiempo libre, relación de pareja y vida familiar). Se divide en 6 ítems que valoran dichos aspectos siguiendo un sistema de puntuación que va de 0 a 5 (nada, casi nada, poco, bastante, mucho y muchísimo). El punto de corte establecido es de 12 en la puntuación total (obtenida sumando los distintos ítems) y de 2 en cada uno de ellos, con una puntuación tanto mayor cuanto mayor es la inadaptación.

\section{Resultados}

\section{Ansiedad y depresión}

Los resultados muestran que la edad media de aparición de los síntomas es de 30 años. La puntuación media de ansiedad entre los encuestados asciende a 7,48 $(D T=3,62)$, mientras que la de depresión es de 6,98 (DT = 4,34). Asimismo, la prevalencia de ansiedad (considerando como tal únicamente la categoría del HADS «Probable problema clínico») en personas que presentan esclerosis múltiple es de un $20 \%$, mientras que la prevalencia en la muestra de personas con un probable proble ma de depresión es de un $21,67 \%$.

Atendiendo al análisis diferencial de la ansiedad y la depresión en función de distintas variables sociodemográficas como el género, la situación laboral, el estado civil, la comunidad autónoma en la que reside el paciente, la edad y la edad de aparición de los síntomas, se observan diferencias significativas solo en función del género (ansiedad: $p$-valor $=0,013$; y depresión: $p$-valor $=0,008$ ), siendo la puntuación media en ansiedad mayor en hombres (media $=9,04 ; \quad D T=3,98$ ) que en mujeres (media $=6,51 ; \mathrm{DT}=3,05)$. De igual forma, la puntuación media en depresión también es mayor en hombres (media $=8,78$; DT $=3,77$ ) que en mujeres (media $=5,87$; DT $=4,34$ ). En la tabla 5 se muestran los p-valores de cada una de las variables consideradas.

Tabla 5

P-valores asociados a las comparaciones de las variables sociodemográficas con la ansiedad y la depresión

\begin{tabular}{|c|c|c|}
\hline Variable & Ansiedad & Depresión \\
\hline $\begin{array}{c}\text { Sexo } \\
\text { Situación } \\
\text { laboral }\end{array}$ & 0,013 & 0,0082 \\
\hline $\begin{array}{c}\text { Estado civil } \\
\text { Edad }\end{array}$ & 0,126 & 0,170 \\
\hline $\begin{array}{c}\text { Edad de } \\
\text { aparición de } \\
\text { síntomas }\end{array}$ & 0,815 & 0,627 \\
\hline $\begin{array}{c}\text { Comunidad } \\
\text { autónoma }\end{array}$ & 0,428 & 0,162 \\
\hline
\end{tabular}


Basándonos en las categorías establecidas por el HADS, podemos observar que el $34,78 \%$ de los hombres se incluyen en la categoría «nada de ansiedad», mientras que un $39,13 \%$ podrían presentar un probable problema de ansiedad. Un $72,97 \%$ de las mujeres se clasificarían en la categoría «nada de ansiedad», mientras que un $8,11 \%$ sí podría presentar un probable problema de ansiedad. En cuanto a la depresión, un $30,43 \%$ de los hombres de la muestra se incluyen en la categoría «nada de depresión» mientras que un $39,13 \%$ podría llegar a tener un probable problema de depresión. Un $67,57 \%$ de las mujeres se incluirían en la categoría «nada de depresión» mientras que un 10,81 \% de las mismas podrían llegar a tener un probable problema de depresión (véanse las tablas 6 y 7 ).

Tabla 6

Frecuencia absoluta y porcentaje de gente que pueden tener o no tener algún tipo de problema clínico con la ansiedad.

\begin{tabular}{|c|c|c|c|c|c|c|c|}
\hline Ansiedad & Nada & Posible & Probable & $\begin{array}{c}\text { Nada } \\
\mathbf{( \% )}\end{array}$ & $\begin{array}{c}\text { Posible } \\
(\mathbf{\%})\end{array}$ & $\begin{array}{c}\text { Probable } \\
(\%)\end{array}$ & Total \\
\hline \hline Hombre & 8 & 6 & 9 & 34,78 & 26,09 & 39,13 & 23 \\
\hline Mujer & 27 & 7 & 3 & 72,97 & 18,92 & 8,11 & 37 \\
\hline Total & 35 & 13 & 12 & 58,33 & 21,67 & 20,00 & 60 \\
\hline
\end{tabular}

Tabla 7

Frecuencia absoluta y porcentaje de gente que puede tener o no tener algún tipo de problema clínico con la depresión.

\begin{tabular}{|l|c|c|c|c|c|c|c|}
\hline Depresión & Nada & Posible & Probable & $\begin{array}{l}\text { Nada } \\
\mathbf{( \% )}\end{array}$ & $\begin{array}{l}\text { Posible } \\
(\mathbf{\%})\end{array}$ & $\begin{array}{l}\text { Probable } \\
\mathbf{( \% )}\end{array}$ & Total \\
\hline Hombre & 7 & 7 & 9 & 30,43 & 30,43 & 39,13 & 23 \\
\hline Mujer & 25 & 8 & 4 & 67,57 & 21,62 & 10,81 & 37 \\
\hline Total & 32 & 15 & 13 & 53,33 & 25,00 & 21,67 & 60 \\
\hline
\end{tabular}

\section{Inadaptación}

En cuanto a la interferencia provocada por la enfermedad en los diferentes ámbitos tenidos en cuenta en la escala de inadaptación, se observa un porcentaje de inadaptados (media superior a 2) considerablemente elevado, alcanzando un 63,33\% de los encuestados, con una puntuación media de 2,45 (DT = 1,33).

Cada uno de los 6 ítems que componen este test proporciona información aislada y específica de una faceta de la vida del individuo y, por lo tanto, pueden y deben ser estudiados de forma separada. En el primero de ellos, que hace referencia al ámbito laboral o académico, observamos que el $66,67 \%$ de los participantes han obtenido puntuaciones por encima de los 2 puntos (lo que se consideraría como inadaptados), siendo la puntuación media de $3,08(D T=1,61)$. En lo referente a la faceta social, hay un $48,33 \%$ de sujetos que superan el umbral de inadaptación con media de 2,22 $(\mathrm{DT}=1,47)$. En el caso del ocio y tiempo libre, la cifra asciende a un $63,33 \%$ con una media de 2,68 (DT = 1,49). Respecto a la interferencia en las relaciones de pareja o en la posibilidad de iniciar una, cuenta con un $36,67 \%$ de individuos afectados, con una media de 2,13 (DT = 1,61). En cuanto a las relaciones familiares, el $31,67 \%$ de los 
pacientes han visto afectada esta área con un valor medio de 1,72 (DT = 1,38). Finalmente, el último ítem que compone la escala de inadaptación evalúa la interferencia que supone la esclerosis múltiple en su vida en general. En este último caso, el $65 \%$ de los participantes refieren que sus vidas se han visto afectadas como consecuencia de la enfermedad, con una media de 2,87 (DT = 1,33).

Una vez estudiadas las puntuaciones resultantes de la escala de inadaptación, se procedió al análisis diferencial de la calificación global media del test y las distintas variables sociodemográficas recogidas. Así pues, se realizaron comparaciones con la comunidad autónoma de procedencia, el género, la situación laboral, el estado civil, la edad y la edad de aparición de los primeros síntomas (véase la tabla 8). Tal como podemos apreciar, las diferencias solo han sido significativas desde el punto de vista estadístico en el caso de la situación laboral $(p$-valor $=0,001)$.

Asimismo, analizando cada una de las áreas de la El en función de las distintas variables sociodemográficas, se obtuvieron diferencias significativas solo en función de la situación laboral en el ámbito social $(p$-valor $=0,0031)$, laboral/académico ( $p$ valor $\left.=1,49 \times 10^{-7}\right)$ y de ocio y tiempo libre $(p$-valor $=0,044)$.

Tabla 8

Puntuación global media de la EI comparada con las diferentes variables sociodemográficas

\begin{tabular}{|c|c|c|c|c|c|c|c|c|c|c|}
\hline $\begin{array}{l}\text { Comunidad } \\
\text { autónoma }\end{array}$ & Aragón & & $\begin{array}{l}\text { Castilla y } \\
\text { León }\end{array}$ & \multicolumn{2}{|c|}{$\begin{array}{l}\text { Comunidad } \\
\text { Valenciana }\end{array}$} & \multicolumn{3}{|c|}{ Galicia } & Murcia & P-valor \\
\hline Inadaptación & 1,9 & & 2,377 & & 688 & & 3,042 & & 2,75 & 0,237 \\
\hline \multicolumn{2}{|l|}{ Género } & \multicolumn{3}{|c|}{ Hombres } & \multicolumn{4}{|c|}{ Mujeres } & \multicolumn{2}{|c|}{ P-valor } \\
\hline \multicolumn{2}{|c|}{ Inadaptación } & \multicolumn{2}{|r|}{2,826} & \multicolumn{5}{|c|}{2,204} & \multicolumn{2}{|c|}{0,075} \\
\hline $\begin{array}{c}\text { Situación } \\
\text { laboral }\end{array}$ & Empleado & \multicolumn{2}{|c|}{ Desempleado } & \multicolumn{3}{|c|}{$\begin{array}{c}\text { Incapacidad } \\
\text { laboral }\end{array}$} & \multicolumn{2}{|c|}{ Jubilado } & Otros & P-valor \\
\hline Inadaptación & 1,988 & \multicolumn{2}{|r|}{1,694} & \multicolumn{3}{|c|}{3,487} & \multicolumn{2}{|c|}{2,556} & 3,11 & 0,001 \\
\hline Estado civil & Soltero & \multicolumn{2}{|c|}{$\begin{array}{c}\text { Soltero } \\
\text { con pareja } \\
\end{array}$} & \multicolumn{2}{|c|}{ Casado } & \multicolumn{3}{|c|}{ Separado } & Otros & P-valor \\
\hline Inadaptación & 3,037 & & 1,852 & & 383 & & 3,833 & & 2,75 & 0,239 \\
\hline Edad & \multirow{2}{*}{\multicolumn{2}{|c|}{ Adolescencia }} & \multicolumn{2}{|c|}{ Adulto joven } & \multicolumn{3}{|c|}{$\begin{array}{l}\text { Adulto } \\
\text { maduro }\end{array}$} & \multicolumn{2}{|c|}{ Senectud } & P-valor \\
\hline Inadaptación & & - & \multicolumn{2}{|c|}{2,521} & \multicolumn{3}{|c|}{2,34} & \multicolumn{2}{|c|}{3,778} & 0,15 \\
\hline $\begin{array}{c}\text { Edad de } \\
\text { aparición de } \\
\text { síntomas } \\
\end{array}$ & \multicolumn{2}{|l|}{ Niñez } & \multicolumn{2}{|c|}{ Adolescencia } & \multicolumn{3}{|c|}{ Adulto joven } & \multicolumn{2}{|c|}{$\begin{array}{l}\text { Adulto } \\
\text { maduro }\end{array}$} & P-valor \\
\hline Inadaptación & \multicolumn{2}{|l|}{1,417} & \multicolumn{2}{|l|}{2,25} & 2,3 & & & 2,8 & 302 & 0,406 \\
\hline
\end{tabular}




\section{Discusión y conclusiones}

\section{Discusión}

\section{Ansiedad y depresión}

En primer lugar, la edad media de aparición de los síntomas de la enfermedad en las personas de nuestra muestra es de 30 años, la cual coincide con la de otros estudios, que la establecen en 30,29 años (Kidd et al. 2017; Arbinaga Ibarzábal 2003). Además, el porcentaje de la muestra estudiada con un probable problema de ansiedad es del $20 \%$, bastante similar al de otros estudios, que lo establecen en un $22 \%$; mientras que, en la población general, el porcentaje de gente afectada por ansiedad es del 4,1\% (Agencia EFE 2017). Como podemos ver, según los datos obtenidos en este estudio, el riesgo de sufrir ansiedad entre el colectivo que tiene esclerosis múltiple es de unas 4,88 veces mayor que entre el de la población general.

El porcentaje de depresión de la muestra analizada es del 21,67\%, menor que el obtenido en otros estudios, que lo establecen en el $31 \%$. Aun así, tomando como referencia los valores obtenidos en este estudio y teniendo en cuenta que el 5,2\% de la población global presenta depresión, se puede decir que los pacientes con esclerosis múltiple presentan una probabilidad 4,17 veces mayor de padecer un probable problema de depresión.

A partir de los datos obtenidos, podemos afirmar que la comunidad autónoma en la que vive una persona afectada de esclerosis no se encuentra relacionada con el sufrimiento de ansiedad, depresión o inadaptación general. Asimismo, en estas variables no parece influir el estado civil, la edad del paciente ni la edad de aparición de los síntomas. Sin embargo, el género sí parece influir en la sintomatología de ansiedad y depresión. En este sentido, diversos estudios muestran diferencias en ansiedad y depresión en función del sexo, siendo más prevalentes los problemas de ansiedad y depresión en el colectivo femenino (Armstrong y Khawaja 2002; Dickstein 2000); sin embargo, los resultados de nuestro estudio no siguen esta premisa, sino que son los hombres quienes presentan más síntomas de ansiedad y depresión. Concretamente, los hombres presentarían una probabilidad de sufrir ansiedad 4,82 veces mayor que las mujeres y una probabilidad 3,62 veces mayor de padecer un probable problema de depresión.

\section{Inadaptación}

En lo referente a la inadaptación, llama la atención que los ámbitos menos perjudicados por la enfermedad son los de tipo afectivo y social, destacando entre ellos el de vida familiar (media $=1,72 ; \mathrm{DT}=1,38$ ). En el resto de campos, casi dos terceras partes se consideran inadaptadas, no siendo una excepción la valoración de la interferencia en la vida en general. La esfera del trabajo o estudios es el área más damnificada de todas.

En relación a las posibles diferencias de la puntuación global media de la escala de inadaptación en función de diversas variables sociodemográficas, no hemos obtenido diferencias significativas en función de la comunidad autónoma, el estado civil, la edad, la edad de aparición de los síntomas y el género. Cabe destacar que podrían haberse encontrado diferencias en función del género en el caso de que se hubiese trabajado con una confianza del $92 \%$. Sin embargo, los test del estudio se han realizado a un nivel de fiabilidad del $95 \%$, asignado de forma arbitraria al comienzo del mismo. 
La única variable sociodemográfica en función de la cual se han obtenido diferencias significativas en la puntuación global media de la escala de inadaptación es la situación laboral. Asimismo, se han obtenido diferencias significativas en la inadaptación en el ámbito social, laboral/académico y de ocio y tiempo libre.

A pesar de haber realizado una búsqueda exhaustiva, no se ha encontrado ningún artículo científico que utilice la escala de inadaptación aplicada a pacientes diagnosticados de esclerosis múltiple, por lo que no ha sido posible realizar una comparación entre los datos de este estudio y los de trabajos precedentes.

Conclusiones

La esclerosis múltiple es una enfermedad neurodegenerativa con un efecto muy negativo en el estilo de vida de las personas que la sufren. Como se ha observado en el trabajo, la prevalencia de la ansiedad, la depresión y la inadaptación es muy elevada respecto a la prevalencia de estas en la población sana. Por ello, consideramos que una atención psicológica adecuada desde el diagnóstico puede ser crucial para mejorar un afrontamiento adaptativo a esta enfermedad con el menor coste posible de sufrimiento emocional. Al mismo tiempo, el análisis diferencial en función de distintas variables sociodemográficas en el grado de afectación emocional e interferencia de la enfermedad puede darnos información valiosa para adaptar mejor la terapia psicológica, en función de las características de cada sujeto.

Si bien este estudio aporta datos interesantes sobre la afectación emocional de los pacientes con EM, no está exento de limitaciones, siendo una de las más importantes el tamaño muestral. Por ello, consideramos el presente trabajo como un estudio preliminar, siendo necesario seguir profundizando sobre las implicaciones psicológicas de la esclerosis múltiple, para poder mejorar la calidad de vida de los pacientes que la padecen.

\section{Referencias bibliográficas}

Agencia EFE. 2017. «En España 2,4 millones de personas sufren depresión, el 5,2 \% de la población». Recuperado de: https://www.efe.com/efe/espana/sociedad/en-espana-2-4-millones-depersonas-sufren-depresion-el-5-la-poblacion/10004-3188014 [Consultado el 30 de julio de 2019].

Arbinaga lbarzábal, Félix. 2003. «Aspectos emocionales y calidad de vida en pacientes con enfermedades desmielinizantes: el caso de la esclerosis múltiple». Anales de Psicología 19(1): 65-74.

Armstrong, Kerry y Nigar G. Khawaja. 2002. "Gender differences in anxiety: An investigation of the symptoms, cognitions, and sensitivity towards anxiety in a nonclinical population». Behavioural and Cognitive Psychotherapy 30: 227-231.

Boeschoten, Rosa E., Annemarie Braamse, Aartjan T. F. Beekman, Pim Cuijpers, Patricia van Oppen, Joost Dekker y Bernard M. J. Uitdehaag. 2016. Prevalence of depression and anxiety in Multiple Sclerosis: A systematic review and metaanalysis. Journal of the Neurological Sciences 372: 331-341.

Dickstein, Leah J. 2000. "Gender differences in mood and anxiety disorders: From bench to bedside: American Psychiatric Press Review of Psychiatry». The American Journal of Psychiatry 157: 1186-1187.

Echeburúa Odriozola, Enrique, Paz de Corral Gargallo y Javier Fernández-Montalvo. 2000. «Escala de inadaptación (El): Propiedades psicométricas en contextos clínicos». Análisis y Modificación de Conducta 26: 325-340.

Kidd, Tara, Nicola Carey, Freda Mold, Sue Westwood, Maria Miklaucich, Emmanouela Konstantara, Annette Sterr y Debbie Cooke. 2017. «A systematic review of the 
effectiveness of self- management interventions in people with multiple sclerosis at improving depression, anxiety and quality of life». PLoS ONE 12(10): e0185931. Recuperado de: https:// doi.org/10.1371/journal.pone.0185931 [Consultado el 30 de julio de 2019].

MedlinePlus. 2017. «Esclerosis múltiple». Recuperado de: https://medlineplus.gov/spanish/multiplesclerosis.html [Consultado el 30 de julio de 2019].

Muñoz San José, Ainoa, Celia Oreja-Guevara, Susana Cebolla Lorenzo, Lidia Carrillo Notario, Beatriz Rodríguez Vega y Carmen Bayón Pérez. 2015. «Intervenciones psicoterapéuticas y psicosociales para el manejo del estrés en esclerosis múltiple: aportación de intervenciones basadas en mindfulness». Neurología 31(2): 113-120.

Rodrigues de Cerqueira, Ana Claudia, Patricia Semionato de Andrade, Jose Maurício Godoy Barreiros, Antônio Lúcio Teixeira y Antonio Egidio Nardi. 2015. «Psychiatric disorders in patients with multiple sclerosis». Comprehensive Psychiatry 63: 10-14.

Zigmond, Anthony S. y R. Phillip Snaith. 1983. «The Hospital Anxiety and Depression Scale». Acta Psychiatrica Scandinavica 67: 361-370. 